\title{
Metamaterial Lensing Devices
}

\author{
Jiangtao $\mathrm{Lv}^{1}$, Ming Zhou ${ }^{1}$, Qiongchan $\mathrm{Gu}^{1}{ }^{1}$, Xiaoxiao Jiang ${ }^{1}$, Yu Ying ${ }^{2}$ and Guangyuan $\mathrm{Si}^{1,3, *}$ \\ 1 College of Information Science and Engineering, Northeastern University, Shenyang 110004, China \\ College of Information \& Control Engineering, Shenyang Jianzhu University, Shenyang 110168, China \\ 3 Melbourne Centre for Nanofabrication, Clayton, Victoria 3168, Australia \\ * Correspondence: guangyuan.si@monash.edu
}

Academic Editor: Xuejun Lu

Received: 15 May 2019; Accepted: 2 July 2019; Published: 4 July 2019

\begin{abstract}
In recent years, the development of metamaterials and metasurfaces has drawn great attention, enabling many important practical applications. Focusing and lensing components are of extreme importance because of their significant potential practical applications in biological imaging, display, and nanolithography fabrication. Metafocusing devices using ultrathin structures (also known as metasurfaces) with superlensing performance are key building blocks for developing integrated optical components with ultrasmall dimensions. In this article, we review the metamaterial superlensing devices working in transmission mode from the perfect lens to two-dimensional metasurfaces and present their working principles. Then we summarize important practical applications of metasurfaces, such as plasmonic lithography, holography, and imaging. Different typical designs and their focusing performance are also discussed in detail.
\end{abstract}

Keywords: metamaterial; nanofocusing; perfect lens; metasurfaces

\section{Introduction}

In recent years, surface plasmons and related devices [1-23] have been thoroughly investigated due to their potentially wide applications in nanophotonics [24-38], biology [39-45], spectroscopy [46-51], and so on. They are capable of manipulating electromagnetic waves [52-62] at the nanometer scale to achieve all-optical integration, providing an effective way to develop smaller, faster and more efficient devices. Surface plasmon resonance is based on the oscillation of electrons formed at the metal-dielectric interface. The resonant wavelength can be continuously tuned from ultraviolet to infrared, enabling various practical devices [63-65]. These devices have gained wide attention and experienced considerable development because they have small dimensions, fast operating speed, and low energy consumption. In particular, lensing and focusing components are widely used in many technologies, such as high-resolution imaging [66-70], nanolithography [71-75], and optical integration [76-79]. However, due to the diffraction limit which means the resolvable feature size is determined by $d=\frac{\lambda}{2 \mathrm{NA}}$ (where $d$ is the resolvable feature size, $\lambda$ is the wavelength of incident light, and NA is the numerical aperture) due to the wave nature of radiation, the imaging resolution of conventional lenses and optical systems is difficult to break through the dimension of half of the incident wavelength. Therefore, it is of great scientific and application significance to break through the diffraction limit and achieve super-resolution focusing and imaging effects. Some information of the subwavelength structure (the object) is hidden in the evanescent wave. The intensity decays rapidly with increasing distance from the object and the attenuation speed is proportional to the spatial frequency. Recently, more metamaterial-enabled technologies have been reported, including computing metastructures [80], near-zero-index wires [81], curvilinear metasurfaces for surface wave manipulation [82], acoustic resonators [83], and so on [84,85].

Although Veselago first proposed a negative index medium in 1968 [86], a new concept of a perfect lens proposed by Pendry [87] started to draw great attention after about 30 years, which enabled 
the scientific community to re-understand and examine the abnormal electromagnetic properties and cloaking effects of metamaterial media. Later, Fang et. al. experimentally demonstrated the superlensing effect using an evanescent wave through a silver film [88]. Then the same group investigated the far-field superlensing effect [89] and proposed a hyperlens design. Conventional optical lenses focus by adjusting the phase of the incident light by varying the thickness of optical materials (e.g., glass). Such lenses are bulky and cumbersome. With the continuous development of micro-nano optics, traditional optical lenses are difficult to meet the requirements of large-scale integration and device miniaturization and functional diversification. Metasurface lenses [90-94] are made of two-dimensional (2D) planar structures that artificially arrange optical antennas with special electromagnetic characteristics in a certain way, which can achieve flexible regulation of amplitude, phase, polarization and other parameters of the incident light. Important applications, such as holographic optics and achromatic lenses, have been triggered. In addition, these 2D planar structures are easier to process and integrate, providing solutions for miniaturization and integration of optical lenses. Here, we review the metamaterial superlensing devices (Section 2) and two-dimensional metasurfaces (Section 3) working in transmission mode from ultraviolet to visible range. We then summarize their important practical applications (Section 4), such as plasmonic lithography, holography, and imaging.

\section{Superlensing Effect and Far-Field Hyperlens}

\subsection{Perfect Lens}

Due to the diffraction limit, the imaging resolution of conventional optical lenses can only reach half of the wavelength of the incident light. By recovering and enhancing the high-frequency evanescent wave carrying detailed information of the object, perfect lenses based on metamaterials with negative refractive indices are expected to break through this optical diffraction limit and achieve super-resolution imaging. Due to the loss of light waves propagating in metal, how to enhance the high-frequency evanescent wave signal more efficiently and convert it into a propagating wave to participate in imaging to better achieve far-field super-resolution imaging and how to further increase the near field ultra-high resolution focused spot depth and reduce the far field focused spot size are the focus of further research on surface plasmon-enabled lenses. Pendry first proposed the perfect lens theoretically using negative index media [87], which can amplify the evanescent waves. A perfect lens with a negative refractive index (negative permittivity and permeability) can focus both propagating and evanescent waves. Different from the traditional optical lens, a superlens utilizes the negative refraction effect of the medium to not only converge the propagation wave of the far-field transmission, but also to recover and enhance the near-field evanescent wave signal.

As shown in Figure 1a, since the metamaterial (artificial medium) has a negative refractive index and an evanescent wave amplification characteristic, the evanescent wave (Fourier component) emitted by the electromagnetic wave source will not only be corrected in phase but also its amplitude will be effectively amplified. This can lead to perfect recovery of the object's information at the image point [95-97], breaking the diffraction limited resolution of traditional lens, and achieving the purpose of super-resolution (sub-wavelength) focusing of electromagnetic waves. Figure $1 \mathrm{~b}$ shows an example of a silver slab lens in operation. Although only the imaginary part of the dielectric function prevents ideal reconstruction, a significantly improved focusing effect can still be realized. 


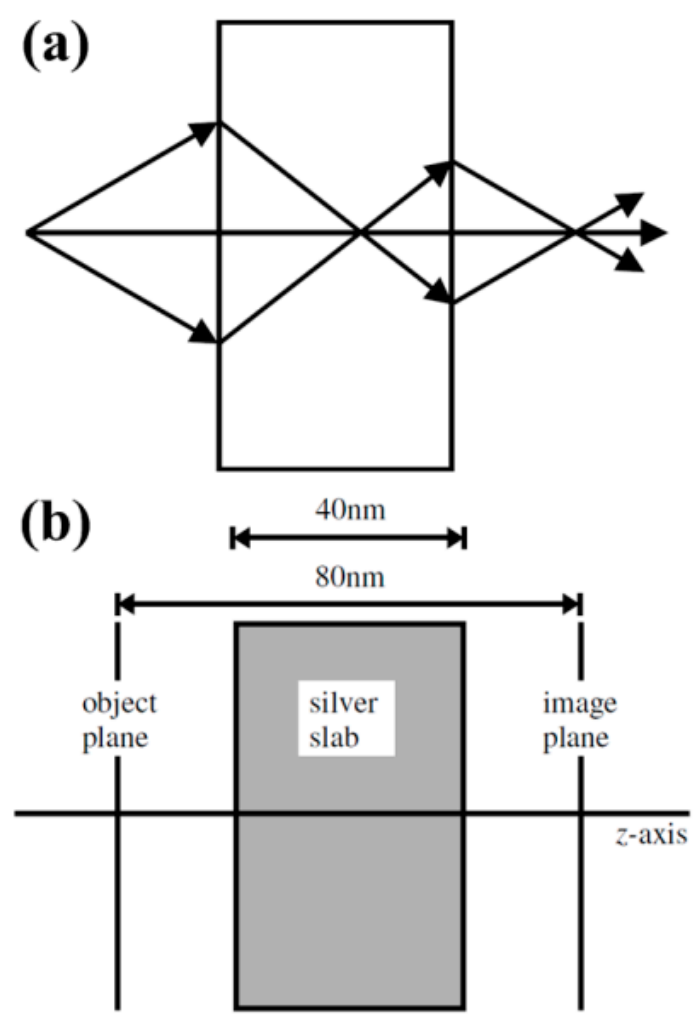

Figure 1. Pendry's perfect lens. (a) Schematic drawing of a parallel-side slab with a negative refractive index acting as a perfect collecting lens. (b) A proposed silver slab lens with dimensions and object/image plane labeled. Reproduced with permission from [87]. Copyright American Physical Society, 2000.

Figure 2 shows a schematic diagram of the super-resolution imaging structure of a silver film superlens developed by Fang and coworkers [88]. Different nanostructures etched in the chromium film by using focused ion beam (FIB) are employed as the imaged objects, and the polymethyl methacrylate (PMMA) layer is used to control the spacing between the chromium film and the silver film superlens. The silver film has a thickness of $35 \mathrm{~nm}$, and the image of the object is recorded on the negative photoresist layer. After development, the pattern of the object image is characterized by atomic force microscopy (AFM). Under the action of incident light with a wavelength of $365 \mathrm{~nm}$, the silver film superlens amplifies the evanescent wave by exciting the surface plasmons, compensating for the exponential decay of the evanescent wave in air propagation, thus realizing super-resolution imaging. As shown in Figure 2c, under the action of the silver film superlens, the objects ("NANO" milled by FIB as shown in Figure 2b) can be clearly identified and the image is sharp and fine. However, when the PMMA layer is used in place of the silver film, the imaging result loses the fine information of the slit array etched on the chromium film, which is blurry as shown in Figure 2d. The silver film superlens achieves a fine imaging width of $89 \mathrm{~nm}(\lambda / 4.1)$, and without the silver film superlens, the imaging width is $(321 \pm 10) \mathrm{nm}$, which is much larger than the imaging size of the former. 

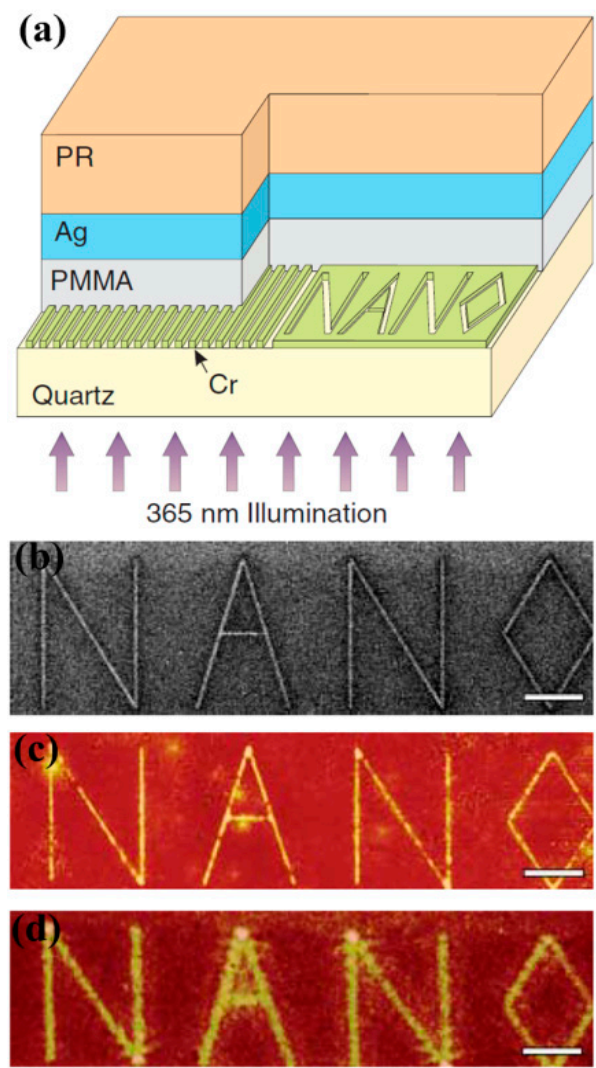

Figure 2. (a) Schematic of a silver superlens. (b) Object of "NANO" milled by FIB. (c) High resolution and fine features with the superlens. (d) Low resolution and blurry features without the superlens. Reproduced with permission from [88]. Copyright the American Association for the Advancement of Science, 2005.

\subsection{Hyperlens with a Far-Field Superlensing Effect}

After achieving the near-field superlensing effect, how to further project the near-field image into the far-field becomes critical. A hyperlens (an anisotropic metamaterial with a hyperbolic dispersion) can realize far-field super-resolution imaging with low losses, which has shown notable potential for real-time biomolecular imaging and nanolithography. Although the silver film can dramatically improve the imaging quality of nanostructures and achieve super-resolution imaging, its working distance is very small and can only be limited to the near field region since the evanescent wave rapidly decays away from the surface of the silver film superlens. Liu et al. further proposed and experimentally verified a hyperlens design that can achieve far-field super-resolution imaging effect [89]. The near-field superlens consists of a single layer of ultra-thin silver film, while the far-field hyperlens adds a periodic grating structure to the ultra-thin silver film, as shown in Figure 3a. The ultra-thin silver film enhances the evanescent wave carrying the fine structure information of the object by exciting the surface plasmon, and then converts the enhanced evanescent wave into a propagating wave by using a periodic grating structure. Figure $3 \mathrm{~b}$ shows the experimental results of a pair of nanoslits with a width of $35 \mathrm{~nm}$ and $150 \mathrm{~nm}$ spacing and their far-field imaging. Conventional optical microscopes without the hyperlens cannot clearly capture the image of the pair of nanoslits. However, when a far-field superlens is used for imaging, the set of nanoslits can be clearly distinguished (Figure $3 \mathrm{~b}$ hyperlens image). It can be seen that surface plasmons play a decisive role in super-resolution imaging of superlenses. Figure 3c shows arbitrary objects (letters of " $\mathrm{O}$ " and " $\mathrm{N}$ ") with $\sim 40 \mathrm{~nm}$ line widths and, again, one can see that sub-diffraction resolution can be obtained. Figure $3 \mathrm{~d}$ plots the averaged cross-section of both hyperlens image and the control case without the hyperlens. 


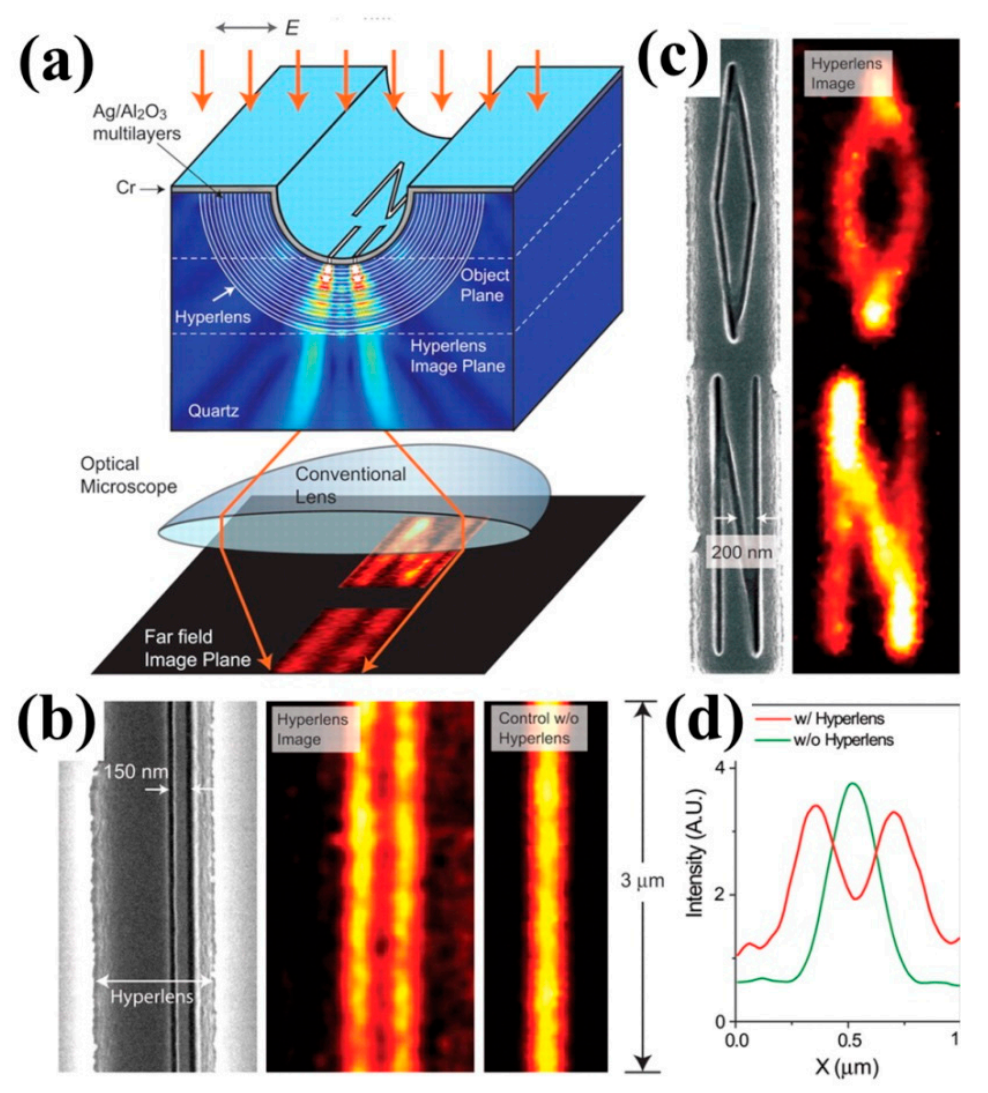

Figure 3. (a) Schematic of the multilayer hyperlens and its working mechanism. (b) SEM image of the slits and far-field images with and without hyperlens. (c) SEM image of letters " $\mathrm{O}$ " and " $\mathrm{N}$ " and far-field image with hyperlens. (d) Averaged cross-section of both hyperlens image and the control case without the hyperlens. Reproduced with permission from [89]. Copyright the American Association for the Advancement of Science, 2007.

\subsection{Planar Lensing with Plasmonic Gratings}

The main limitation for current superlens and hyperlens designs is that most of them need very complex designs and some of them have very limited functional wavelength range. Simple designs with dynamic engineering functionalities are desirable. To simplify lens designs with acceptable cost and test their working principles, different analytical models have been used to verify the superlensing mechanism, such as finite difference time domain (FDTD) method and finite element method (FEM). However, these commercial software packages have inevitable limitations and disadvantages such as inherent mismatch due to frequency dispersive and time discretization [98]. Therefore, researchers have tried different analytical methods including surface wave modeling [99], Green's function formulation [100], polarizability [101], and scattering [102]. Such kinds of analytical models have their own advantages over the commercial packages since researcher can customize their calculations according to various designs. Furthermore, some of them can be readily applied to varying plasmonic devices, providing guidance for fabrication and experimental verification. Using simple plasmonic gratings, one can easily achieve the focusing effect. For instance, Verslegers and coworkers fabricated a planar lens formed by slits with different widths etched in a $400 \mathrm{~nm}$ thick gold film [103]. Phase delay is introduced by varying gap widths and therefore focusing capability can be realized by elaborately arranging the slits. The curved phase front is realized for the transmitted light wave. As shown in Figure $4 \mathrm{a}, 3-\mu \mathrm{m}$-long slits are defined with $80 \mathrm{~nm}$ width in the center to $150 \mathrm{~nm}$ on the side symmetrically. Figure $4 \mathrm{~b}, \mathrm{c}$ show measured and calculated results of metafocusing capability of nanoslits superlens which agree well with each other. The experimental result measured by a confocal microscope equipped with a stepper motor ( $60 \mathrm{~nm}$ resolution in the $\mathrm{z}$ direction) show focusing distance of $5 \mu \mathrm{m}$ which is a 
little bit larger than FDTD simulations. Note that the wavelength is $637 \mathrm{~nm}$ for both measurements and simulations. Moreover, active lensing manipulation [104] can be readily achieved by filling the nanoslits with nonlinear media. The focal length can be simply engineered by controlling the intensity of incident light.
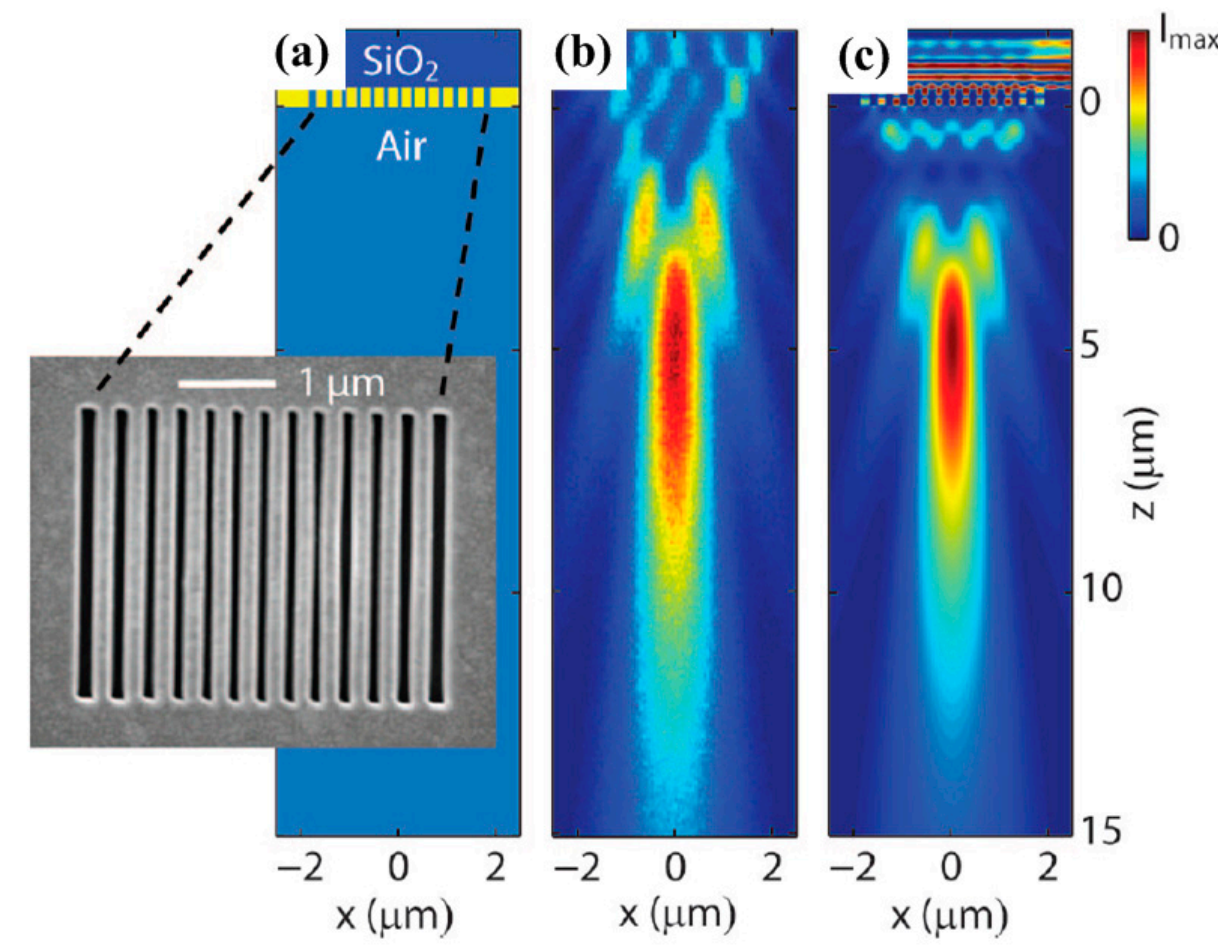

Figure 4. (a) Schematic and SEM image of the nanoslit array plasmonic lens. (b) Experimental results of transmitted intensity distribution captured by a confocal microscope. (c) FDTD-calculated result showing the intensity distribution which agrees well with measurements. Reproduced with permission from [103]. Copyright American Chemical Society, 2009.

\section{Focusing via Metasurfaces}

In this section, we review recent interesting focusing devices triggered by metasurfaces which have been well studied and the most important advantage of these metasurface-enabled devices over bulky optical components is that they are highly convenient for integration with electronics. Furthermore, these thin metasurface designs can generate geometric phase changes and enable more interesting artificial properties that natural materials do not possess. The basic working mechanism is that gradient metasurfaces can introduce space-variant phase (Pancharatnam-Berry phase) changes and, therefore, they can provide many new features [105-109] and, thus, enable more functional applications in optics and photonics. In addition, they could find wide applications in advanced sensing and diagnostics [110], equivalent-circuit model designs [111], and manipulating visible light [112]. More interesting electromagnetic phenomena such as electric currents and surface waves have also been reported [113-115], which have affected the metadevice radiation properties significantly.

\subsection{D Metasurface Lens}

V-shaped nanoantennae metasurfaces were first reported by Yu and coworkers [116]. After that, various designs have been investigated including fish-bone antennae [117-119] and rotating rectangular antennae [120-122]. Lin and coworkers demonstrated a multifunctional metasurface lens which can focus light with different wavelengths to a shared focal plane [123]. As shown in Figure 5a, this so-called interleaved metasurface contains various sub-element designs and, therefore, enables multiple functions within a shared aperture. Experimental results (Figure 5b) show that three different wavelengths can 
focus to a shared focal plane which is around $100 \mu \mathrm{m}$ behind the metasurface lens. Moreover, it is worth to note that the working principles can be readily applicable to any high-index materials at desired frequencies.

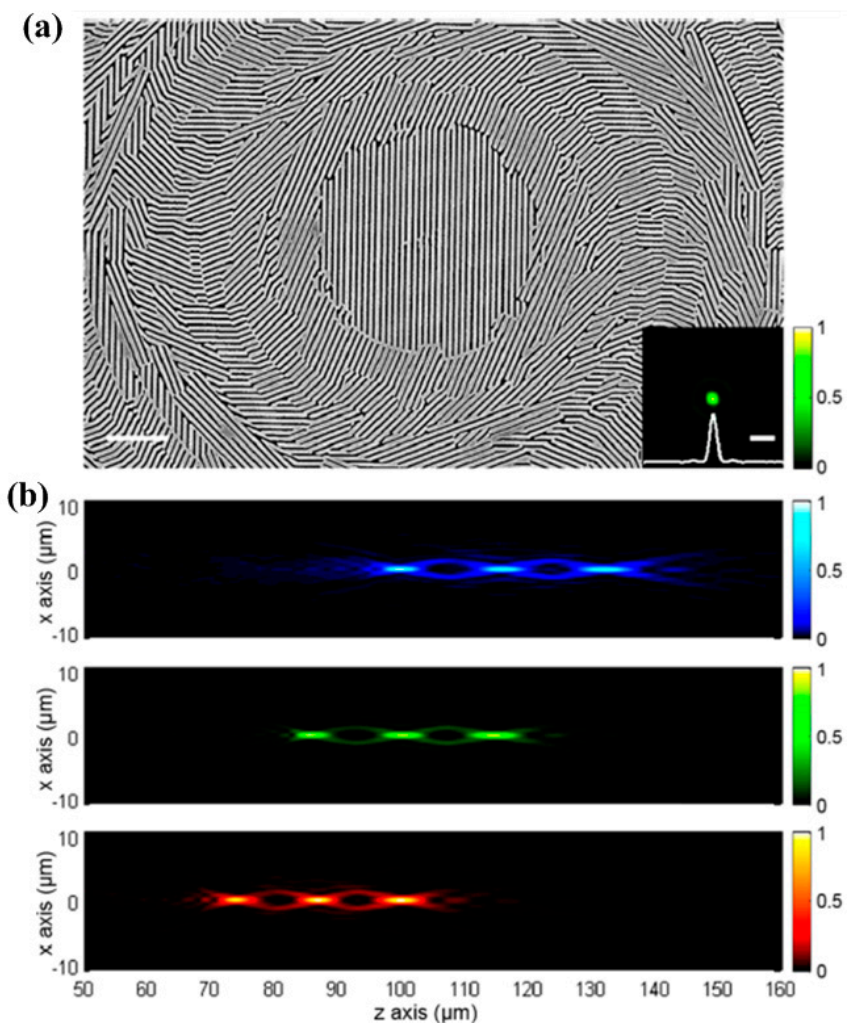

Figure 5. (a) SEM topography of the interleaved metasurface containing various sub-elements. (b) Measured transmitted intensity profiles for blue, green, and red colors. Reproduced with permission from [123]. Copyright American Chemical Society, 2016.

The measured intensity distributions (Figure 5b) also indicate that the transmitted profiles show different focal spots for various colors (red, green, and blue). This interleaved metasurface is essentially an optical antenna array with different geometrical structures and sub-wavelength spatial spacing [124-126]. By designing the phase delay of the radiation field of each nanoantenna, the Huygens principle can be used to achieve arbitrary regulation of the outgoing wavefront. Devices enabled by metasurfaces can achieve precise control of the amplitude, polarization and phase of the incident light field at a scale much smaller than the incident wavelength, which significantly reduces the loss of light propagation in the medium.

\subsection{Dynamic Metasurface Lens with Stretchable Substrates}

Furthermore, a reconfigurable metasurface lens has been experimentally demonstrated with stretchable polydimethylsiloxane (PDMS) substrates [127]. The refraction angle can be manipulated by stretching the substrate mechanically, as shown in Figure 6a. Using electron beam lithography and stripping, a flat lens design is fabricated in PDMS, as shown in Figure 6b. To demonstrate the tunable effect, the longitudinal profiles are reconstructed and the focal length difference can be clearly observed in Figure 6c. Note that such kinds of tunable metasurfaces can regulate the light field with sub-wavelength pixels, which can effectively avoid crosstalk of multi-level diffraction in traditional diffractive optical elements, and they have broad application prospects in micro-integrated photovoltaic systems. In addition, they can perform the same modulation on the light field in a wide band with weak dispersion. These advantages, combined with the superior design flexibility and low-cost manufacturing advantages of metasurfaces, make it a new generation of ultra-thin, ultrasmall-scale 
light field control devices. These devices can be applied to different wavelengths, leading to a universal component. In short, such kind of metalenses can be readily integrated into many commercially available optical devices.

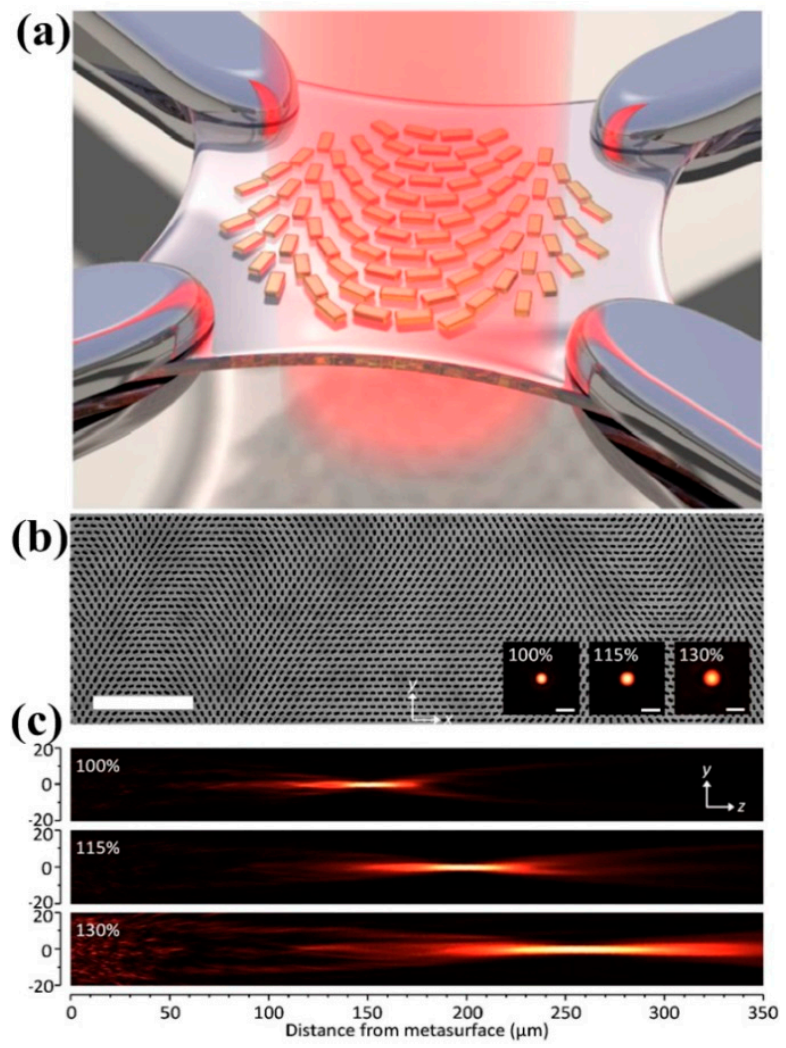

Figure 6. (a) Schematic illustration of a metalens array on a stretchable substrate and (b) SEM image of the metalens array fabricated by EBL and stripping. (c) Measured longitudinal beam profiles generated on the transmission side of the flat zoom lens with different stretch ratios (100\%, $115 \%$, and $130 \%)$. Note that the metasurface is located at the position of $\mathrm{z}=0$. Reproduced with permission from [127]. Copyright American Chemical Society, 2016.

\subsection{Near-Field Fish-Bone Metalens}

To further investigate the near field properties of metasurfaces, Spektor and coworkers [128] employed fishbone-shaped nanoantennae and characterized the near-field properties using a scanning near-field optical microscope (SNOM). Fishbone structures were first studied by Lin and coworkers [117] and it was found that the propagation direction could be controlled by the polarization state of the incident light. Different propagation directions can be realized with varying incident beam (circularly polarized light). Using a spiral metasurface design as shown in Figure 7a, one can achieve high efficiency and uniformity focusing function with circularly polarized illumination. Figure $7 \mathrm{~b}$ plots the phase as a function of the polarization orientation and one can clearly see a linear phase dependence for linear polarizations. Under circularly polarized illumination, however, selective focusing function reveals as shown in Figure 7c (right circularly polarized) and Figure 7e (left circularly polarized), which agree well with FDTD calculations as shown in Figure 7d,f, respectively. 


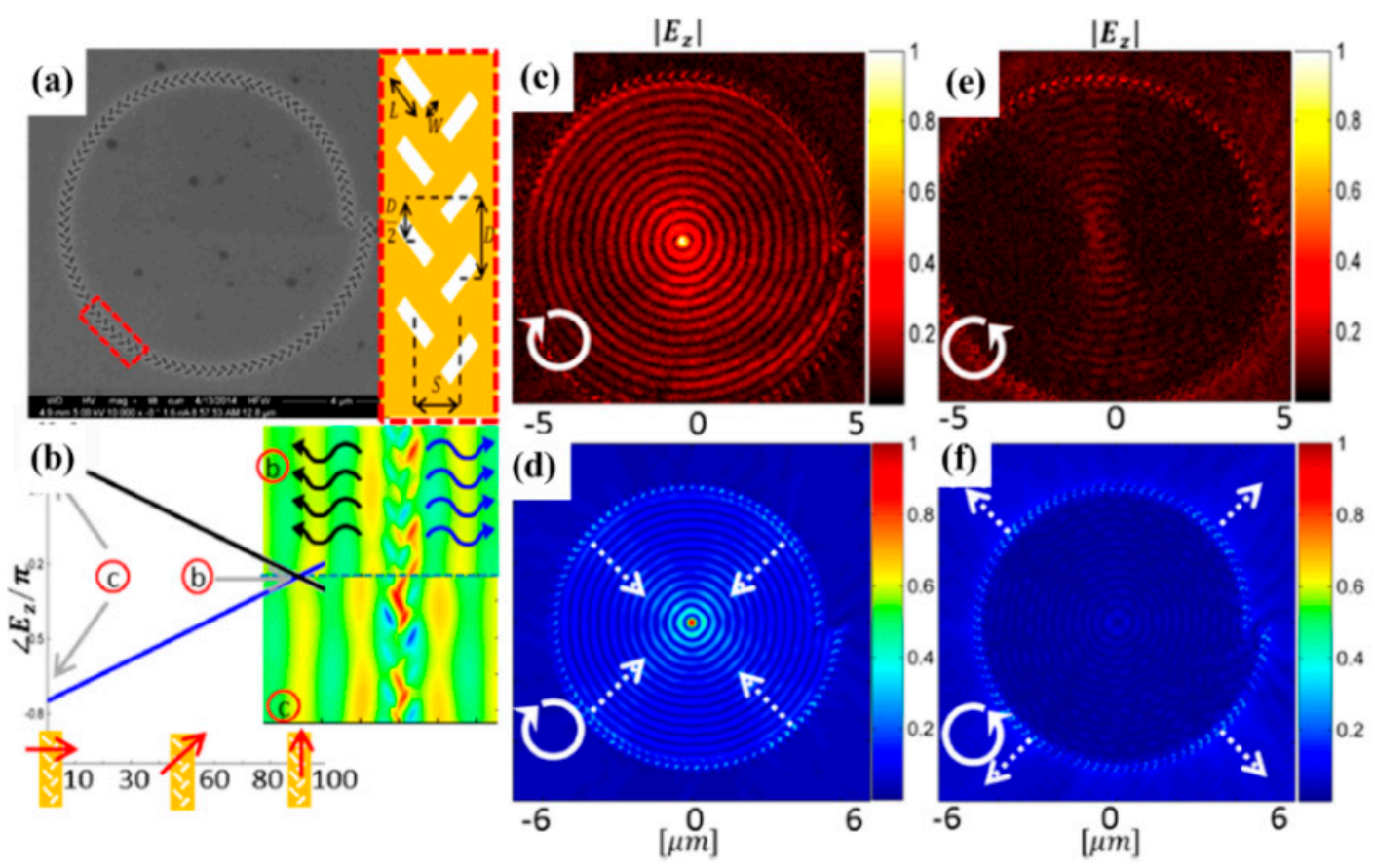

Figure 7. (a) SEM and schematic illustration of the fishbone structures. (b) Linear phase dependence of surface plasmon polaritons as a function of the polarization orientation. (c) Experimental SNOM and (d) calculated results for right circularly polarized incidence. (e) Experimental SNOM and (f) calculated results for left circularly polarized incidence. Reproduced with permission from [128]. Copyright American Chemical Society, 2015.

\section{Potential Practical Applications}

\subsection{Flying Lens for Nanolithography}

Figure 8 a shows the schematic drawing of a maskless nanolithography system which combines a plasmonic flying head and a rotating substrate [129]. By using an air-bearing slider under ultraviolet illumination, a low-cost and high-throughput nanofabrication technique can be achieved. Laser pulses are manipulated by an optical modulator and the writing position is accurately dominated by a spindle encoder. The spinning substrate can produce a so-called air bearing surface which can provide an aerodynamic lift force, enabling accurate regulation of the nanogap. Further experimental results [130] using a multistage plasmonic lens array, as shown in Figure $8 b, c$, with a $355 \mathrm{~nm}$ wavelength illumination show that $22 \mathrm{~nm}$ half pitch resolution can be realized at $7 \mathrm{~m} / \mathrm{s}$ substrate velocity and $160 \mathrm{MHz}$ laser pulse repetition rate. This kind of low-cost and high-throughput direct writing approach may enable new nanolithography techniques with high speed and high aspect ratio [131]. Moreover, it may be useful for magnetic data storage technology (e.g., heat-assisted magnetic recording) to achieve larger capacities. 

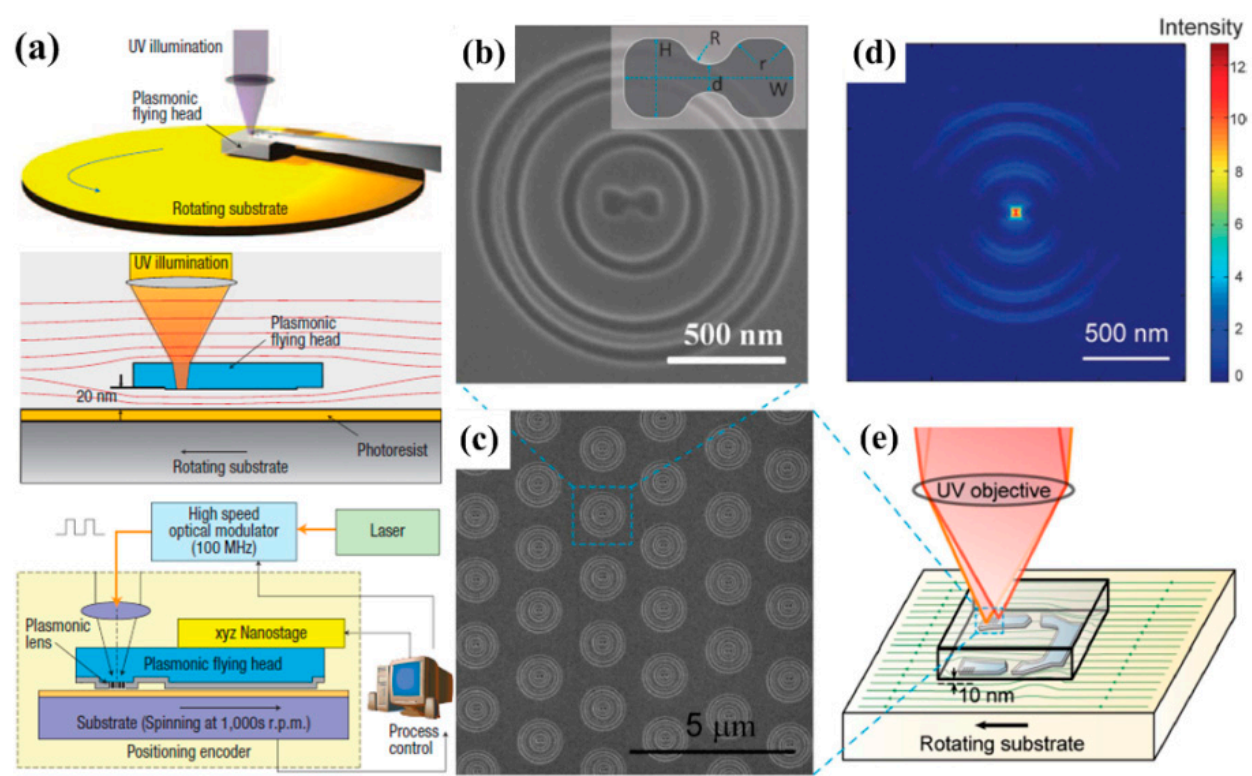

Figure 8. (a) Schematic of the maskless nanolithography concept using a flying lens. (b) Magnified SEM and (c) overview of the plasmonic lens structure. (d) Calculated intensity distribution at $10 \mathrm{~nm}$ distance away from the lens with $355 \mathrm{~nm}$ incident wavelength. (e) Schematic of the plasmonic flying head using advanced airbearing surface. Reproduced with permission from [129] and [130]. Copyright Springer Nature, 2008 and 2011.

\subsection{Metalens for Fluorescence Imaging}

Another important application of a metalens is for biological imaging thanks to their unique capability of wavefront shaping. Most recently, Jang and co-workers experimentally demonstrated a disorder-engineered metalens which can enable the individual input-output responses [132]. Note that the disordered metalens is composed of silicon nitride $\left(\mathrm{SiN}_{\mathrm{x}}\right)$ nanoposts which can function as truncated multimode waveguides. Moreover, wavefront shaping using a disorder-engineered metalens can enable access to a wide optical space and stable optical focusing function with high-quality.

Figure 9a shows the low-resolution bright-field image of immunofluorescence-labeled parasites using a conventional fluorescence microscope with a $4 \times$ objective lens and one can see that the magnified image shown in Figure $9 d$ is blurry. Figure $9 b, e, f$ present the images obtained with a metalens at different locations of $(x, y)=(0,0),(1,1)$ and $(2.5,0)$, respectively. One can see that fine features can be resolved with the capability for high numerical aperture focusing. Figure $9 \mathrm{c}$ is the ground-truth fluorescence image captured with a $20 \times$ objective lens for comparison. More useful application examples include biomedical sensing [133,134], electromagnetic wave absorbing [135], active beam switching and bifocal zoom lensing [136], telecommunications [137], and so on [138,139]. Most of these metalens-enabled components can be readily used and applied to various optical devices and act as functional instruments which can significantly help improve the device performance. Furthermore, one should note that the new functionalities introduced in this review of these metalensing devices possess high integration compatibility with electronics as well due to their small dimensions, leading to more practical miniaturization designs for optics and nanophotonics. 

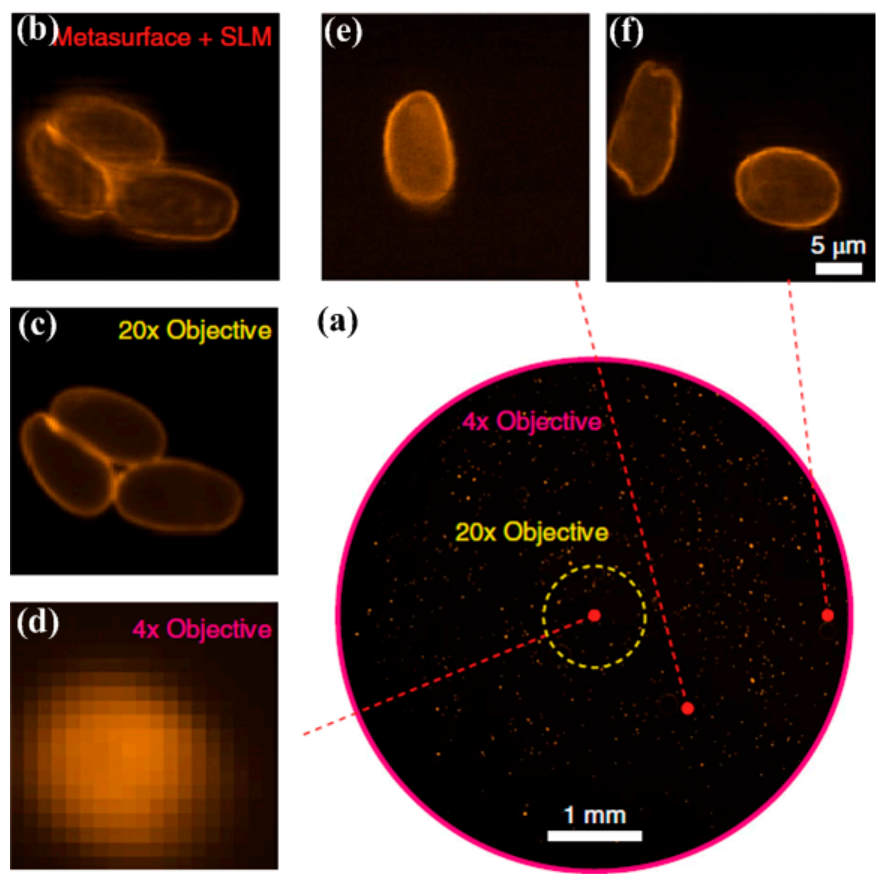

Figure 9. (a) Low-resolution image captured by a conventional fluorescence microscope with a $4 \times$ objective lens. (b) Scanned image obtained with a disordered metalens. (c) Ground-truth fluorescence image captured with a $20 \times$ objective lens. (d) Magnified low-resolution fluorescence image captured with a $4 \times$ objective lens. (e, f) Images obtained with the disorder-metalens assisted microscope at $(x, y)$ $=(1,1)$ and $(2.5,0) \mathrm{mm}$, respectively. Reproduced with permission from [132]. Copyright Springer Nature, 2018.

\section{Conclusions and Outlook}

To conclude, we have reviewed the development of superlensing and metafocusing devices and their potential applications. Due to the recent development of metasurfaces, as well as the exploration of new optical functions, dynamic plasmonic lensing devices and more practical achievements have been developed, paving the way for new plasmonic imaging and focusing technologies. The current developing trends for actively tunable metalens seem to be towards higher modulating speed and large-scale manufacturing or even massive production. However, researchers still need to investigate the feasibility to achieve higher resolution and better optical performance since the main limitations of the metadevices are still challenging, which may need great efforts and investigations, such as developing adaptive metalenses, which can manipulate focal length, astigmatism, and shift simultaneously [140]. In addition, highly tunable devices with low losses are of extreme importance since they may enable more innovations due to their exceptional capability of manipulating electromagnetic waves at the sub-wavelength scale. More future demands and improvements have been proposed including optical modulators, radiative cooling metasurfaces, and new dispersionless flat lenses [114]. Miniaturization of optical metalensing devices will also become an important trend [141] due to their ultrasmall dimensions.

Author Contributions: Conceptualization, J.L. and G.S.; investigation, J.L., M.Z. and Q.G.; investigation, X.J.; resources, Y.Y.; writing-original draft preparation, J.L.; writing-review and editing, G.S.; visualization, X.J.; supervision, G.S.; funding acquisition, G.S.

Funding: We thank the National Natural Science Foundation of China (grant No. 11704263), the Natural Science Foundation of Hebei Province (grant No. F2018501063), Hebei Province Science and Technology Plan Key Research and Development Project Research Funds (grant No. 18273902D) for financial support. This work was performed in part at the Melbourne Centre for Nanofabrication $(\mathrm{MCN})$ in the Victorian Node of the Australian National Fabrication Facility (ANFF).

Conflicts of Interest: The authors declare no conflict of interest. 


\section{References}

1. Halas, N.J.; Lal, S.; Chang, W.S.; Link, S.; Nordlander, P. Plasmons in strongly coupled metallic nanostructures. Chem. Rev. 2011, 111, 3913-3961. [CrossRef] [PubMed]

2. Lv, J.; Leong, E.S.P.; Jiang, X.; Kou, S.; Dai, H.; Lin, J.; Liu, Y.J.; Si, G. Plasmon-enhanced sensing: Current status and prospects. J. Nanomater. 2015, 2015, 474730. [CrossRef]

3. Si, G.; Jiang, X.; Lv, J.; Gu, Q.; Wang, F. Fabrication and characterization of well-aligned plasmonic nanopillars with ultrasmall separations. Nanoscale Res. Lett. 2014, 9, 299. [CrossRef] [PubMed]

4. Lv, J.; Li, Z.; Ying, Y.; Yang, L.; Yang, N.; Jiang, X.; Si, G. Recent progress on metasurface-enabled plasmonics. Nanosci. Nanotech. Lett. 2015, 7, 779-786. [CrossRef]

5. Si, G.; Wang, Q.; Lv, J.; Miao, L.; Wang, F.; Peng, S. Interference lithography patterned large area plasmonic nanodisks for infrared detection. Mater. Lett. 2014, 128, 373-375. [CrossRef]

6. Yap, F.L.; Thoniyot, P.; Krishnan, S.; Krishnamoorthy, S. Nanoparticle cluster arrays for high-performance SERS through directed self-assembly on flat substrates and on optical fibers. ACS Nano 2012, 6, 2056-2070. [CrossRef] [PubMed]

7. Lv, J.; Gu, Q.; Jiang, X.; Yang, L.; Li, Z.; Ma, Z.; Si, G. Plasmonic nanoantennae with ultrasmall gaps and their application in surface enhanced Raman scattering. Nanosci. Nanotech. Lett. 2015, 7, 917-919. [CrossRef]

8. Tian, C.F.; Ding, C.H.; Liu, S.Y.; Yang, S.C.; Song, X.P.; Ding, B.J.; Li, Z.Y.; Fang, J.X. Nanoparticle attachment on silver corrugated-wire nanoantenna for large increases of surface-enhanced Raman scattering. ACS Nano 2011, 5, 9442-9449. [CrossRef]

9. Fazio, B.; D’Andrea, C.; Bonaccorso, F.; Irrera, A.; Calogero, G.; Vasi, C.; Gucciardi, P.G.; Allegrini, M.; Toma, A.; Chiappe, D.; et al. Re-radiation enhancement in polarized surface-enhanced resonant Raman scattering of randomly oriented molecules on self-organized gold nanowires. ACS Nano 2011, 5, 5945-5956. [CrossRef]

10. Si, G.; Zhao, Y.; Lv, J.; Wang, F.; Liu, H.; Teng, J.; Liu, Y.J. Direct and accurate patterning of plasmonic nanostructures with ultrasmall gaps. Nanoscale 2013, 5, 4309-4313. [CrossRef]

11. Jones, M.R.; Osberg, K.D.; Macfarlane, R.J.; Langille, M.R.; Mirkin, C.A. Templated techniques for the synthesis and assembly of plasmonic nanostructures. Chem. Rev. 2011, 111, 3736-3827. [CrossRef] [PubMed]

12. Wang, J.; Jiang, X.; Xia, L.; Tang, L.; Hu, S.; Lv, J.; Zhao, H.; Si, G.; Shi, R. Fabrication and optical measurement of double-overlapped annular apertures. Opt. Mater. 2016, 60, 13-16. [CrossRef]

13. Liu, J.; He, H.; Xiao, D.; Yin, S.; Ji, W.; Jiang, S.; Luo, D.; Wang, B.; Liu, Y. Recent advances of plasmonic Nnanoparticles and their applications. Materials 2018, 11, 1833. [CrossRef] [PubMed]

14. Si, G.; Zhao, Y.; Lv, J.; Lu, M.; Wang, F.; Liu, H.; Xiang, N.; Huang, T.J.; Danner, A.J.; Teng, J.; et al. Reflective plasmonic color filters based on lithographically patterned silver nanorod arrays. Nanoscale 2013, 5, 6243-6248. [CrossRef] [PubMed]

15. Kuznetsov, A.I.; Evlyukhin, A.B.; Goncalves, M.R.; Reinhardt, C.; Koroleva, A.; Arnedillo, M.L.; Kiyan, R.; Marti, O.; Chichkov, B.N. Laser fabrication of large-scale nanoparticle arrays for sensing applications. ACS Nano 2011, 5, 4843-4849. [CrossRef]

16. Leong, E.S.P.; Deng, J.; Khoo, E.H.; Wu, S.; Phua, W.K.; Liu, Y.J. Fabrication of suspended, three-dimensional chiral plasmonic nanostructures with single-step electron-beam lithography. RCS Adv. 2015, 5, 96366-96371. [CrossRef]

17. Si, G.; Leong, E.S.P.; Pan, W.; Chum, C.C.; Liu, Y.J. Plasmon-induced transparency in coupled triangle-rod arrays. Nanotechnology 2014, 26, 025201. [CrossRef]

18. Leong, E.S.P.; Liu, Y.J.; Deng, J.; Fong, Y.T.; Zhang, N.; Wu, S.J.; Teng, J.H. Fluid-enabled significant enhancement and active tuning of magnetic resonances in free-standing plasmonic metamaterials. Nanoscale 2014, 6, 11106-11111. [CrossRef]

19. Christensen, J.; Manjavacas, A.; Thongrattanasiri, S.; Koppens, F.H.L.; de Abajo, F.J.G. Graphene plasmon waveguiding and hybridization in individual and paired nanoribbons. ACS Nano 2012, 6, 431-440. [CrossRef]

20. Wang, W.; Yang, Q.; Fan, F.; Xu, H.; Wang, Z.L. Light propagation in curved silver nanowire plasmonic waveguides. Nano Lett. 2011, 11, 1603-1608. [CrossRef]

21. Si, G.; Zhao, Y.; Leong, E.S.P.; Lv, J.; Liu, Y.J. Incident-angle dependent color tuning from a single plasmonic chip. Nanotechnology 2014, 25, 455203. [CrossRef] [PubMed] 
22. Smolyaninov, I.I.; Hung, Y.-J.; Davis, C.C. Magnifying superlens in the visible frequency range. Science 2007, 315, 1699-1701. [CrossRef] [PubMed]

23. Lv, J.; Jiang, X.; Ying, Y.; Si, G. Controlling light through a refined superlens. Nanosci. Nanotech. Lett. 2015, 7, 901-905. [CrossRef]

24. Cai, W.; Chettiar, U.K.; Kildishev, A.V.; Shalaev, V.M. Optical cloaking with metamaterials. Nat. Photon. 2007, 1, 224-227. [CrossRef]

25. Wegener, M.; Dolling, G.; Linden, S. Backward waves moving forward. Nat. Mater. 2007, 6, 475-476. [CrossRef]

26. Lv, J.-T.; Yan, Y.; Zhang, W.-K.; Liu, Y.-H.; Jiang, Z.-Y.; Si, G.-Y. Plasmonic nanoantennae fabricated by focused ion beam milling. Int. J. Precis. Eng. Man. 2015, 16, 851-855. [CrossRef]

27. Chong, K.E.; Staude, I.; James, A.; Dominguez, J.; Liu, S.; Campione, S.; Subramania, G.; Luk, T.S.; Decker, M.; Neshev, D.N.; et al. Polarization-independent silicon metadevices for efficient optical wavefront control. Nano Lett. 2015, 15, 5369-5374. [CrossRef]

28. Lv, J.; Wang, F.; Ma, Z.; Si, G. Nanoring color filters based on Fabry-Pérot cavities. Acta Phys. Sin. 2013, 62, 057804 .

29. Dickson, W.; Wurtz, G.A.; Evans, P.; O’Connor, D.; Atkinson, R.; Pollard, R.; Zayats, A.V. Dielectric-loaded plasmonic nanoantenna arrays: A metamaterial with tunable optical properties. Phys. Rev. B 2007, 76, 115411. [CrossRef]

30. Wurtz, G.A.; Pollard, R.; Zayats, A.V. Optical bistability in nonlinear surface-plasmon polaritonic crystals. Phys. Rev. Lett. 2006, 97, 057402. [CrossRef]

31. Lv, J.; Khoo, E.H.; Leong, E.S.P.; Hu, L.; Jiang, X.; Li, Y.; Luo, D.; Si, G.; Liu, Y.J. Maskless fabrication of slanted annular aperture arrays. Nanotechnology 2017, 28, 225302. [CrossRef] [PubMed]

32. Jiang, X.; Leong, E.S.P.; Liu, Y.J.; Si, G. Tuning plasmon resonance in depth-variant plasmonic nanostructures. Mater. Des. 2016, 96, 64-67. [CrossRef]

33. Soukoulis, C.M.; Wegener, M. Past achievements and future challenges in the development of three-dimensional photonic metamaterials. Nat. Photon. 2011, 5, 523-530. [CrossRef]

34. Jiang, X.; Gu, Q.; Yang, L.; Zhao, R.; Lv, J.; Ma, Z.; Si, G. Functional plasmonic crystal nanoantennae with ultrasmall gaps and highly tunable profiles. Opt. Laser Technol. 2015, 71, 1-5. [CrossRef]

35. Xiao, D.; Liu, Y.J.; Yin, S.; Liu, J.; Ji, W.; Wang, B.; Luo, D.; Li, G.; Sun, X.W. Liquid-crystal-loaded chiral metasurfaces for reconfigurable multiband spin-selective light absorption. Opt. Express 2018, 26, 25305-25314. [CrossRef]

36. Jiang, X.; Hu, S.; Li, Z.; Lv, J.; Si, G. Fabrication and characterization of plasmonic nanorods with high aspect ratios. Opt. Mater. 2016, 58, 323-326. [CrossRef]

37. Si, G.; Zhao, Y.; Leong, E.S.P.; Liu, Y.J. Liquid-crystal-enabled active plasmonics: A review. Materials 2014, 7 , 1296-1317. [CrossRef]

38. Kang, B.; Woo, J.H.; Choi, E.; Lee, H.H.; Kim, E.S.; Kim, J.; Hwang, T.J.; Park, Y.S.; Kim, D.H.; Wu, J.W. Optical switching of near infrared light transmission in metamaterial-liquid crystal cell structure. Opt. Express 2010, 18, 16492-16498. [CrossRef]

39. Nguyen, H.H.; Park, J.; Kang, S.; Kim, M. Surface plasmon resonance: A versatile technique for biosensor applications. Sensors 2015, 15, 10481-10510. [CrossRef]

40. Lakowicz, J.R. Plasmonics in biology and plasmon-controlled fluorescence. Plasmonics 2016, 1, 5-33. [CrossRef]

41. Nath, N.; Chilkoti, A. Label-free biosensing by surface plasmon resonance of nanoparticles on glass: optimization of nanoparticle size. Anal. Chem. 2004, 76, 5370-5378. [CrossRef] [PubMed]

42. Cao, S.; Shao, Y.; Wang, Y.; Wu, T.; Zhang, L.; Huang, Y.; Zhang, F.; Liao, C.; He, J.; Wang, Y. Highly sensitive surface plasmon resonance biosensor based on a low-index polymer optical fiber. Opt. Express 2018, 26, 3988-3994. [CrossRef]

43. Gorodkiewicz, E.; Lukaszewski, Z. Recent progress in surface plasmon resonance biosensors (2016 to Mid-2018). Biosensors 2018, 8, 132. [CrossRef] [PubMed]

44. Yang, C.-T.; Xu, Y.; Pourhassan-Moghaddam, M.; Tran, D.P.; Wu, L.; Zhou, X.; Thierry, B. Surface plasmon enhanced light scattering biosensing: Size dependence on the gold nanoparticle tag. Sensors 2019, 19, 323. [CrossRef] [PubMed] 
45. Wang, D.; Loo, J.F.C.; Chen, J.; Yam, Y.; Chen, S.-C.; He, H.; Kong, S.K.; Ho, H.P. Recent advances in surface plasmon resonance imaging sensors. Sensors 2019, 19, 1266. [CrossRef] [PubMed]

46. Pryce, I.M.; Kelaita, Y.A.; Aydin, K.; Atwater, H.A. Compliant metamaterials for resonantly enhanced infrared absorption spectroscopy and refractive index sensing. ACS Nano 2011, 5, 8167-8174. [CrossRef] [PubMed]

47. Wu, C.H.; Khanikaev, A.B.; Adato, R.; Arju, N.; Yanik, A.A.; Altug, H.; Shvets, G. Fano-resonant asymmetric metamaterials for ultrasensitive spectroscopy and identification of molecular monolayers. Nat. Mater. 2012, 11, 69-75. [CrossRef] [PubMed]

48. Anh, N.D.; Chun, B.J.; Choi, S.; Kim, D.-E.; Kim, S.; Kim, Y.-J. Plasmonic dynamics measured with frequency-comb-referenced phase spectroscopy. Nat. Phys. 2019, 15, 132-137. [CrossRef]

49. Costa, J.S.; Zaman, Q.; da Costa, K.Q.; Dmitriev, V.; Pandoli, O.; Fontes, G.; Del Rosso, T. Limits of the effective medium theory in particle amplified surface plasmon resonance spectroscopy biosensors. Sensors 2019, 19, 584. [CrossRef]

50. Li, Y.; Bai, H.; Zhai, J.; Yi, W.; Li, J.; Yang, H.; Xi, G. Alternative to noble metal substrates: Metallic and plasmonic $\mathrm{Ti}_{3} \mathrm{O}_{5}$ hierarchical microspheres for surface enhanced Raman spectroscopy. Anal. Chem. 2019, 91, 4496-4503. [CrossRef]

51. Tsur, Y.; Arie, A. On-chip plasmonic spectrometer. Opt. Lett. 2016, 41, 3523-3526. [CrossRef] [PubMed]

52. Shelby, R.A.; Smith, D.R.; Schultz, S. Experimental verification of a negative index of refraction. Science 2001, 292, 77-79. [CrossRef] [PubMed]

53. Rogacheva, A.V.; Fedotov, V.A.; Schwanecke, A.S.; Zheludev, N.I. Giant gyrotropy due to electromagnetic-field coupling in a bilayered chiral structure. Phys. Rev. Lett. 2006, 97, 177401. [CrossRef] [PubMed]

54. Earl, S.K.; James, T.D.; Gomez, D.E.; Marvel, R.E.; Haglund, R.F.; Roberts, A. Switchable polarization rotation of visible light using a plasmonic metasurface. APL Photon. 2016, 2, 016103. [CrossRef]

55. Hao, J.; Yuan, Y.; Ran, L.; Jiang, T.; Kong, A.; Chan, C.T.; Zhou, L. Manipulating electromagnetic wave polarizations by anisotropic metamaterials. Phys. Rev. Lett. 2007, 99, 063908. [CrossRef]

56. Plum, E.; Liu, X.X.; Fedotov, V.A.; Chen, Y.; Tsai, D.P.; Zheludev, N.I. Metamaterials: Optical activity without chirality. Phys. Rev. Lett. 2009, 102, 113902. [CrossRef] [PubMed]

57. Fedotov, V.A.; Rogacheva, A.V.; Zheludev, N.I.; Mladyonov, P.L.; Prosvirnin, S.L. Mirror that does not change the phase of reflected waves. Appl. Phys. Lett. 2006, 88, 091119. [CrossRef]

58. Landy, N.I.; Sajuyigbe, S.; Mock, J.J.; Smith, D.R.; Padilla, W.J. Perfect metamaterial absorber. Phys. Rev. Lett. 2008, 100, 207402. [CrossRef]

59. Fedotov, V.A.; Mladyonov, P.L.; Prosvirnin, S.L.; Rogacheva, A.V.; Chen, Y.; Zheludev, N.I. Asymmetric propagation of electromagnetic waves through a planar chiral structure. Phys. Rev. Lett. 2006, 97, 167401. [CrossRef]

60. Menzel, C.; Helgert, C.; Rockstuhl, C.; Kley, E.B.; Tunnermann, A.; Pertsch, T.; Lederer, F. Asymmetric transmission of linearly polarized light at optical metamaterials. Phys. Rev. Lett. 2010, 104, 253902. [CrossRef]

61. Novitsky, A.V.; Galynsky, V.M.; Zhukovsky, S.V. Asymmetric transmission in planar chiral split-ring metamaterials: Microscopic Lorentz-theory approach. Phys. Rev. B 2012, 86, 075138. [CrossRef]

62. Schurig, D.; Mock, J.J.; Justice, B.J.; Cummer, S.A.; Pendry, J.B.; Starr, A.F.; Smith, D.R. Metamaterial electromagnetic cloak at microwave frequencies. Science 2006, 314, 977-980. [CrossRef] [PubMed]

63. Monticone, F.; Alù, A. Metamaterial, plasmonic and nanophotonic devices. Rep. Prog. Phys. 2017, 80, 036401. [CrossRef] [PubMed]

64. Sun, J.; Litchinitser, N.M. Toward practical, subwavelength, visible-light photolithography with hyperlens. ACS Nano 2018, 12, 542-548. [CrossRef] [PubMed]

65. Yu, X.; Yuan, Y.; Xu, J.; Yong, K.-T.; Qu, J.; Song, J. Strong coupling in microcavity structures: Principle, design, and practical application. Laser Photonics Rev. 2019, 13, 1800219. [CrossRef]

66. Nguyen, H.A.; Banerjee, P.; Nguyen, D.; Lyding, J.W.; Gruebele, M.; Jain, P.K. STM imaging of localized surface plasmons on individual gold nanoislands. J. Phys. Chem. Lett. 2018, 9, 1970-1976. [CrossRef] [PubMed]

67. Simoncelli, S.; Li, Y.; Cortes, E.; Maier, S.A. Imaging plasmon hybridization of Fano resonances via hot-electron-mediated absorption mapping. Nano Lett. 2018, 18, 3400-3406. [CrossRef] [PubMed]

68. Banville, F.A.; Moreau, J.; Sarkar, M.; Besbes, M.; Canva, M.; Charette, P.G. Spatial resolution versus contrast trade-off enhancement in high-resolution surface plasmon resonance imaging (SPRI) by metal surface nanostructure design. Opt. Express 2018, 26, 10616-10630. [CrossRef] 
69. Stravers, C.S.; Gool, E.L.; van Leeuwen, T.G.; Aalders, M.C.G.; van Dam, A. Multiplex body fluid identification using surface plasmon resonance imaging with principal component analysis. Sens. Actuators B Chem. 2019, 283, 355-362. [CrossRef]

70. Tumkur, T.; Yang, X.; Zhang, C.; Yang, J.; Zhang, Y.; Naik, G.V.; Nordlander, P.; Halas, N.J. Wavelengthdependent optical force imaging of bimetallic Al-Au heterodimers. Nano Lett. 2018, 18, 2040-2046. [CrossRef]

71. Jiang, Z.; Luo, H.; Guo, S.; Wang, L. $40 \mathrm{~nm}$ thick photoresist-compatible plasmonic nanolithography using a bowtie aperture combined with a metal-insulator-metal structure. Opt. Lett. 2019, 44, 783-786. [CrossRef] [PubMed]

72. Luo, Y.; Jiang, X.; Liu, L.; Si, G. Recent advances in plasmonic nanolithography. Nanosci. Nanotech. Lett. 2018, 10, 1-12. [CrossRef]

73. Kong, W.; Luo, Y.; Zhao, C.; Liu, L.; Gao, P.; Pu, M.; Wang, C.; Luo, X. Plasmonic interference lithography for low-cost fabrication of dense lines with sub-50 nm half-pitch. ACS Appl. Nano Mater. 2019, 2, 489-496. [CrossRef]

74. Lin, L.; Li, J.; Li, W.; Yogeesh, M.N.; Shi, J.; Peng, X.; Liu, Y.; Rajeeva, B.B.; Becker, M.F.; Liu, Y.; et al. Optothermoplasmonic nanolithography for on-demand patterning of 2D materials. Adv. Funct. Mater. 2018, 28, 1803990. [CrossRef]

75. Kim, T.; Lee, W.-S.; Joe, H.-E.; Lim, G.; Choi, G.-J.; Gang, M.-G.; Kang, S.-M.; Park, K.-S.; Min, B.-K.; Park, Y.-P.; et al. High-speed plasmonic nanolithography with a solid immersion lens-based plasmonic optical head. Appl. Phys. Lett. 2012, 101, 161109. [CrossRef]

76. Zeng, Y.-S.; Qu, S.-W.; Chen, B.-J.; Chan, C.H. All-plasmonic optical phased array integrated on a thin-film platform. Sci. Rep. 2017, 7, 9959. [CrossRef] [PubMed]

77. Wang, C.; Zhang, M.; Chen, X.; Bertrand, M.; Shams-Ansari, A.; Chandrasekhar, S.; Winzer, P.; Lončar, M. Integrated lithium niobate electro-optic modulators operating at CMOS-compatible voltages. Nature 2018, 562, 101-104. [CrossRef] [PubMed]

78. Shi, X.; Verschueren, D.; Pud, S.; Dekker, C. Integrating sub-3 nm plasmonic gaps into solid-state nanopores. Small 2017, 14, 1703307. [CrossRef]

79. Harter, T.; Muehlbrandt, S.; Ummethala, S.; Schmid, A.; Nellen, S.; Hahn, L.; Freude, W.; Koos, C. Silicon-plasmonic integrated circuits for terahertz signal generation and coherent detection. Nat. Photon. 2018, 12, 625-633. [CrossRef]

80. Estakhri, N.M.; Edwards, B.; Engheta, N. Inverse-designed metastructures that solve equations. Science 2019, 363, 1333-1338. [CrossRef]

81. La Spada, L.; Vegni, L. Near-zero-index wires. Opt. Express 2017, 25, 23699-23708. [CrossRef] [PubMed]

82. La Spada, L.; Spooner, C.; Haq, S.; Hao, Y. Curvilinear metasurfaces for surface wave manipulation. Sci. Rep. 2019, 9, 3107. [CrossRef] [PubMed]

83. Lee, I.-H.; Yoo, D.; Avouris, P.; Low, T.; Oh, S.-H. Graphene acoustic plasmon resonator for ultrasensitive infrared spectroscopy. Nat. Nanotechnol. 2019, 14, 313-319. [CrossRef] [PubMed]

84. La Spada, L.; Vegni, L. Electromagnetic nanoparticles for sensing and medical diagnostic applications. Materials 2018, 11, 603. [CrossRef] [PubMed]

85. Greybush, N.J.; Pacheco-Pena, V.; Engheta, N.; Murray, C.B.; Kagan, C.R. Plasmonic optical and chiroptical response of self-assembled Au nanorod equilateral trimers. ACS Nano 2019, 13, 1617-1624. [CrossRef] [PubMed]

86. Veselago, V.G. The electrodynamics of substances with simultaneously negative values of $\varepsilon$ and $\mu$. Sov. Phys. Usp. 1968, 10, 509-514. [CrossRef]

87. Pendry, J.B. Negative refraction makes a perfect lens. Phys. Rev. Lett. 2000, 85, 3966-3969. [CrossRef] [PubMed]

88. Fang, N.; Lee, H.; Sun, C.; Zhang, X. Sub-diffraction-limited optical imaging with a silver superlens. Science 2005, 308, 534-537. [CrossRef] [PubMed]

89. Liu, Z.; Lee, H.; Xiong, Y.; Sun, C.; Zhang, X. Far-field optical hyperlens magnifying sub-diffraction-limited objects. Science 2007, 315, 1686. [CrossRef] [PubMed]

90. Zhang, Z.; Wen, D.; Zhang, C.; Chen, M.; Wang, W.; Chen, S.; Chen, X. Multifunctional light sword metasurface lens. ACS Photon. 2018, 5, 1794-1799. [CrossRef]

91. Yang, C.; Shen, Y.; Xie, Y.; Zhou, Q.; Deng, X.; Cao, J. Terahertz planar lenses based on plasmonic metasurfaces. Phys. Lett. A 2019, 383, 789-792. [CrossRef] 
92. Lu, D.Y.; Cao, X.; Wang, K.J.; He, M.D.; Wang, D.; Li, J.; Zhang, X.M.; Liu, L.; Luo, J.H.; Li, Z.; et al. Broadband reflective lens in visible band based on aluminum plasmonic metasurface. Opt. Express 2018, 26, 34956-34964. [CrossRef] [PubMed]

93. Papaioannou, M.; Plum, E.; Rogers, E.T.F.; Zheludev, N.I. All-optical dynamic focusing of light via coherent absorption in a plasmonic metasurface. Light Sci. Appl. 2018, 7, 17157. [CrossRef] [PubMed]

94. Blechman, Y.; Almeida, E.; Sain, B.; Prior, Y. Optimizing the nonlinear optical response of plasmonic metasurfaces. Nano Lett. 2019, 19, 261-268. [CrossRef] [PubMed]

95. Anzan-Uz-Zaman, M.; Song, K.; Lee, E.; Hur, S. In-depth study on resonant tunneling for subwavelength imaging. Sci. Rep. 2018, 8, 15535. [CrossRef]

96. Ma, C.; Kim, S.; Fang, N.X. Far-field acoustic subwavelength imaging and edge detection based on spatial filtering and wave vector conversion. Nat. Commun. 2019, 10, 204. [CrossRef]

97. Haghtalab, M.; Safavi-Naeini, S. Freeform engineered disordered metalenses for super-resolution imaging and communication. Opt. Express 2018, 26, 9749-9771. [CrossRef]

98. Chen, J.J.; Grzegorczyk, T.M.; Wu, B.-I.; Kong, J.A. Limitation of FDTD in simulation of a perfect lens imaging system. Opt. Express 2005, 13, 10840-10845. [CrossRef]

99. La Spada, L.; Haq, S.; Hao, Y. Modeling and design for electromagnetic surface wave devices. Radio Sci. 2017, 52, 1049-1057. [CrossRef]

100. Vegni, L.; Cicchetti, R.; Capece, P. Spectral dyadic Green's function formulation for planar integrated structures. IEEE Trans. Antennas Propag. 1988, 36, 1057-1065. [CrossRef]

101. La Spada, L.; McManus, T.M.; Dyke, A.; Haq, S.; Zhang, L.; Cheng, Q.; Hao, Y. Surface wave cloak from graded refractive index nanocomposites. Sci. Rep. 2016, 6, 29363. [CrossRef] [PubMed]

102. Pacheco-Peña, V.; Engheta, N.; Kuznetsov, S.; Gentselev, A.; Beruete, M. All-Metallic Epsilon-Near-Zero Graded-Index Converging Lens at Terahertz Frequencies. In Proceedings of the 12th European Conference on Antennas and Propagation (EuCAP 2018), London, UK, 9-13 April 2018.

103. Verslegers, L.; Catrysse, P.B.; Yu, Z.; White, J.S.; Barnard, E.S.; Brongersma, M.L.; Fan, S. Planar lenses based on nanoscale slit arrays in a metallic film. Nano Lett. 2009, 9, 235-238. [CrossRef] [PubMed]

104. Min, C.; Wang, P.; Jiao, X.; Deng, Y.; Ming, H. Beam manipulating by metallic nano-optic lens containing nonlinear media. Opt. Express 2007, 15, 9541-9546. [CrossRef] [PubMed]

105. Ghasemi, M.; Choudhury, P.K. Nanostructured concentric gold ring resonator-based metasurface filter device. Optik 2016, 127, 9932-9936. [CrossRef]

106. Giman, F.N.; Soh, P.J.; Jamlos, M.F.; Lago, H.; Al-Hadi, A.A.; Abdulmalek, M.; Abdulaziz, N. Conformal dual-band textile antenna with metasurface for WBAN application. Appl. Phys. A 2017, 123, 32. [CrossRef]

107. Huang, Y.; Yang, L.; Li, J.; Wang, Y.; Wen, G. Polarization conversion of metasurface for the application of wide band low-profile circular polarization slot antenna. Appl. Phys. Lett. 2016, 109, 054101. [CrossRef]

108. Shen, Z.; Jin, B.; Zhao, J.; Feng, Y.; Kang, L.; Xu, W.; Chen, J.; Wu, P. Design of transmission-type coding metasurface and its application of beam forming. Appl. Phys. Lett. 2016, 109, 121103. [CrossRef]

109. Dong, T.; Ma, X.; Mittra, R. Modeling large nonuniform optical antenna arrays for metasurface application. J. Appl. Phys. 2013, 114, 043103. [CrossRef]

110. La Spada, L. Metasurfaces for advanced sensing and diagnostics. Sensors 2019, 19, 355. [CrossRef]

111. Baena, J.D.; Bonache, J.; Martin, F.; Sillero, R.M.; Falcone, F.; Lopetegi, T.; Laso, M.A.G.; Garcia-Garcia, J.; Gil, I.; Portillo, M.F.; et al. Equivalent-circuit models for split-ring resonators and complementary split-ring resonators coupled to planar transmission lines. IEEE Trans. Microw. Theory Tech. 2005, 53, 1451-1461. [CrossRef]

112. Qin, F.; Ding, L.; Zhang, L.; Monticone, F.; Chum, C.C.; Deng, J.; Mei, S.; Li, Y.; Teng, J.; Hong, M.; et al. Hybrid bilayer plasmonic metasurface efficiently manipulates visible light. Sci. Adv. 2016, 2, e1501168. [CrossRef] [PubMed]

113. Tretyakov, S.A. Metasurfaces for general transformations of electromagnetic fields. Phil. Trans. R. Soc. A 2015, 373, 20140362. [CrossRef] [PubMed]

114. Chen, H.T.; Taylor, A.J.; Yu, N. A review of metasurfaces: Physics and applications. Rep. Prog. Phys. 2016, 79, 076401. [CrossRef] [PubMed]

115. Achouri, K.; Caloz, C. Space-wave routing via surface waves using a metasurface system. Sci. Rep. 2018, 8, 7549. [CrossRef] [PubMed] 
116. Yu, N.; Genevet, P.; Kats, M.A.; Aieta, F.; Tetienne, J.P.; Capasso, F.; Gaburro, Z. Light propagation with phase discontinuities: Generalized laws of reflection and refraction. Science 2011, 334, 333-337. [CrossRef]

117. Lin, J.; Mueller, J.P.B.; Wang, Q.; Yuan, G.; Antoniou, N.; Yuan, X.C.; Capasso, F. Polarization-controlled tunable directional coupling of surface plasmon polaritons. Science 2013, 340, 331-334. [CrossRef] [PubMed]

118. Du, L.; Kou, S.S.; Balaur, E.; Cadusch, J.J.; Roberts, A.; Abbey, B.; Yuan, X.C.; Tang, D.; Lin, J. Broadband chirality-coded meta-aperture for photon-spin resolving. Nat. Commun. 2015, 6, 10051. [CrossRef]

119. Rauter, P.; Lin, J.; Genevet, P.; Khanna, S.P.; Lachab, M.; Davies, A.G.; Linfield, E.H.; Capasso, F. Electrically pumped semiconductor laser with monolithic control of circular polarization. Proc. Natl. Acad. Sci. USA 2014, 111, E5623-E5632. [CrossRef]

120. Zhang, L.; Liu, S.; Li, L.; Cui, T.J. Spin-controlled multiple pencil beams and vortex beams with different polarizations generated by Pancharatnam-Berry coding metasurfaces. ACS Appl. Mater. Interfaces 2017, 9, 36447-36455. [CrossRef]

121. Kim, M.; Wong, A.M.H.; Eleftheriades, G.V. Optical Huygens' metasurfaces with independent control of the magnitude and phase of the local reflection coefficients. Phys. Rev. X 2014, 4, 041042. [CrossRef]

122. Bar-David, J.; Levy, U. Nonlinear diffraction in asymmetric dielectric metasurfaces. Nano Lett. 2019, 19, 1044-1051. [CrossRef] [PubMed]

123. Lin, D.; Holsteen, A.L.; Maguid, E.; Wetzstein, G.; Kik, P.G.; Hasman, E.; Brongersma, M.L. Photonic multitasking interleaved Si nanoantenna phased array. Nano Lett. 2016, 16, 7671-7676. [CrossRef] [PubMed]

124. Lin, D.; Fan, P.; Hasman, E.; Brongersma, M.L. Dielectric gradient metasurface optical elements. Science 2014, 345, 298-302. [CrossRef] [PubMed]

125. Lin, D.; Holsteen, A.L.; Maguid, E.; Fan, P.; Kik, P.G.; Hasman, E.; Brongersma, M.L. Polarization-independent metasurface lens employing the Pancharatnam-Berry phase. Opt. Express 2018, 26, 24835-24842. [CrossRef] [PubMed]

126. Holsteen, A.L.; Lin, D.; Kauvar, I.; Wetzstein, G.; Brongersma, M.L. A light-field metasurface for high-resolution single-particle tracking. Nano Lett. 2019, 19, 2267-2271. [CrossRef]

127. Ee, H.-S.; Agarwal, R. Tunable metasurface and flat optical zoom lens on a stretchable substrate. Nano Lett. 2016, 16, 2818-2823. [CrossRef]

128. Spektor, G.; David, A.; Gjonaj, B.; Bartal, G.; Orenstein, M. Metafocusing by a metaspiral plasmonic lens. Nano Lett. 2015, 15, 5739-5743. [CrossRef]

129. Srituravanich, W.; Pan, L.; Wang, Y.; Sun, C.; Bogy, D.B.; Zhang, X. Flying plasmonic lens in the near field for high-speed nanolithography. Nat. Nanotechnol. 2008, 3, 733-737. [CrossRef]

130. Pan, L.; Park, Y.; Xiong, Y.; Ulin-Avila, E.; Wang, Y.; Zeng, L.; Xiong, S.; Rho, J.; Sun, C.; Bogy, D.B.; et al. Maskless plasmonic lithography at $22 \mathrm{~nm}$ resolution. Sci. Rep. 2011, 1, 175. [CrossRef]

131. Gao, P.; Yao, N.; Wang, C.; Zhao, Z.; Luo, Y.; Wang, Y.; Gao, G.; Liu, K.; Zhao, C.; Luo, X. Enhancing aspect profile of half-pitch $32 \mathrm{~nm}$ and $22 \mathrm{~nm}$ lithography with plasmonic cavity lens. Appl. Phys. Lett. 2015, 106, 093110. [CrossRef]

132. Jang, M.; Horie, Y.; Shibukawa, A.; Brake, J.; Liu, Y.; Kamali, S.M.; Arbabi, A.; Ruan, H.; Faraon, A.; Yang, C. Wavefront shaping with disorder-engineered metasurfaces. Nat. Photon. 2018, 12, 84-90. [CrossRef] [PubMed]

133. Pacheco-Peña, V.; Beruete, M.; Rodríguez-Ulibarri, P.; Engheta, N. On the performance of an ENZ-based sensor using transmission line theory and effective medium approach. New J. Phys. 2019, 21, 043056. [CrossRef]

134. Iovine, R.; La Spada, L.; Vegni, L. Optical properties of modified nanorod particles for biomedical sensing. IEEE Trans. Magn. 2014, 50, 169-172. [CrossRef]

135. La Spada, L.; Vegni, L. Metamaterial-based wideband electromagnetic wave absorber. Opt. Express 2016, 24, 5763-5772. [CrossRef] [PubMed]

136. Yin, X.; Steinle, T.; Huang, L.; Taubner, T.; Wuttig, M.; Zentgraf, T.; Geissen, H. Beam switching and bifocal zoom lensing using active plasmonic metasurfaces. Light Sci. Appl. 2017, 6, e17016. [CrossRef] [PubMed]

137. Jin, Y.; Djafari-Rouhani, B.; Torrent, D. Gradient index photonic crystals and metamaterials. Nanophotonics 2019, 8, 685-701. [CrossRef]

138. Li, Y.; Engheta, N. Capacitor-inspired metamaterial inductors. Phys. Rev. Appl. 2018, 10, 054021. [CrossRef]

139. Shaltout, A.M.; Kim, J.; Boltasseva, A.; Shalaev, V.M.; Kildishev, A. Ultrathin and multicolour optical cavities with embedded metasurfaces. Nat. Commun. 2018, 9, 2673. [CrossRef] 
140. She, A.; Zhang, S.; Shian, S.; Clarke, D.R.; Capasso, F. Adaptive metalenses with simultaneous electrical control of focal length, astigmatism, and shift. Sci. Adv. 2018, 4, eaap9957. [CrossRef]

141. Liu, B.; Song, K.; Xiao, J. Two-dimensional optical metasurfaces: From plasmons to dielectrics. Adv. Cond. Matter Phys. 2019, 2019, 2329168. [CrossRef] 